\title{
Strengthening Surgery Strengthens Health Systems: A New Paradigm and Potential Pathway for Horizontal Development in Low- and Middle-Income Countries
}

\author{
S. Ariane Christie ${ }^{1} \cdot$ Benedict C. Nwomeh $^{2} \cdot$ Sanjay Krishnaswami $^{3} \cdot$ George P. Yang $^{4} \cdot$ \\ Ai-Xuan L. Holterman ${ }^{5}$ Anthony Charles ${ }^{6} \cdot$ Sudha Jayaraman $^{7} \cdot$ Randeep S. Jawa $^{8}$. \\ Jennifer Rickard ${ }^{9} \cdot$ Mamta Swaroop $^{10} \cdot$ Sifri C. Ziad ${ }^{11}$ - Georges Alain Etoundi Mballa ${ }^{12}$. \\ Martin Ekeke Monono $^{13} \cdot$ Alain Chichom Mefire ${ }^{14} \cdot$ Catherine Juillard $^{1}$
}

Published online: 15 November 2018

\begin{abstract}
Global health is transitioning toward a focus on building strong and sustainable health systems in developing countries; however, resources, funding, and agendas continue to concentrate on "vertical" (disease-based) improvements in care. Surgical care in low- and middle-income countries (LMICs) requires the development of health systems infrastructure and can be considered an indicator of overall system readiness. Improving surgical care provides a scalable gateway to strengthen health systems in multiple domains. In this position paper by the Society of University Surgeons' Committee on Global Academic Surgery, we propose that health systems development appropriately falls within the purview of the academic surgeon. Partnerships between academic surgical institutions and societies from high-income and resource-constrained settings are needed to strengthen advocacy and funding efforts and support development of training and research in LMICs.
\end{abstract}

Catherine Juillard

Catherine.Juillard@ucsf.edu

1 UCSF Center for Global Surgical Studies, Zuckerberg San Francisco General Hospital and Trauma Center, 1001 Potrero Avenue, Ward 3A, San Francisco, CA 94110, USA

2 Ohio State University Wexner Medical Center, 700 Childrens Dr., Columbus, OH 43205, USA

3 Oregon Health and Science University Medical Center, 3181 SW Sam Jackson Park Rd \#300, Portland, OR 97239, USA

4 Department of Surgery, University of Alabama at Birmingham, 1900 University Blvd., THT Room 1028, Birmingham, AL 35233, USA

5 Department of Pediatrics and Surgery, University of Illinois College of Medicine, 840 S. Wood St., MC856, Chicago, IL 60612, USA

6 University of North Carolina School of Medicine, 4008 Burnett Womack Building CB 7228, Chapel Hill, NC, USA

7 VCU Program for Global Surgery, Department of Surgery, VCU School of Medicine, Box 980454, Richmond, VA 23298-0454, USA
8 Department of Surgery, Stony Brook University School of Medicine, Health Sciences Center T18-040, Stony Brook Medicine, Stony Brook, NY 11794-8191, USA

9 Department of Surgery and Critical Care, University of Minnesota, 420 Delaware Street SE, Mayo Mail Code 195, Minneapolis, MN 55455, USA

10 Northwestern University's Feinberg School of Medicine, 676 North St Clair, Suite 650, Chicago, IL 60611, USA

11 Rutgers New Jersey Medical School, 90 Bergen St, Newark, NJ 07103, USA

12 Ministry of Public Health, Cameroon, Boulevard Rudolph Manga Bell, Yaoundé, Cameroon

13 World Health Organization (WHO) Regional Office, PO Box 06, Brazzaville, Republic of the Congo

14 Faculty of Health Sciences, University of Buea, Molyko, Buea, Cameroon 


\section{Introduction}

Historically, global health has prioritized the treatment of communicable diseases. Even today, the majority of global research and advocacy funding supports programs targeting specific infectious diseases like malaria, tuberculosis, and human immunodeficiency virus (HIV) [1]. Viewed as highimpact, low-cost interventions, treatment of communicable diseases has generally demonstrated high efficacy and led to decreased global mortality over the past decades [2].

The successes of these programs at the same time as ongoing economic growth have led to an "epidemiologic transition" in many LMICs (Fig. 1). Non-communicable disease (NCDs) and injuries make up a large proportion of the global disease burden and should receive greater prioritization and investment by researchers and policymakers $[3,4]$. In particular, injury and surgical care are gaining traction as important areas for development as multiple studies have demonstrated that improving surgical capacity is a cost-effective means of reducing disability and mortality in LMICs [5-7].

However, unlike disease-specific programs, the ability to deliver quality surgical care relies on development of a mature health system and improving surgical infrastructure inherently results in the strengthening of health systems as a whole. As such, in this consensus position by the Society of University Surgeons' Committee on Global Academic Surgery, we propose (1) surgical delivery should be

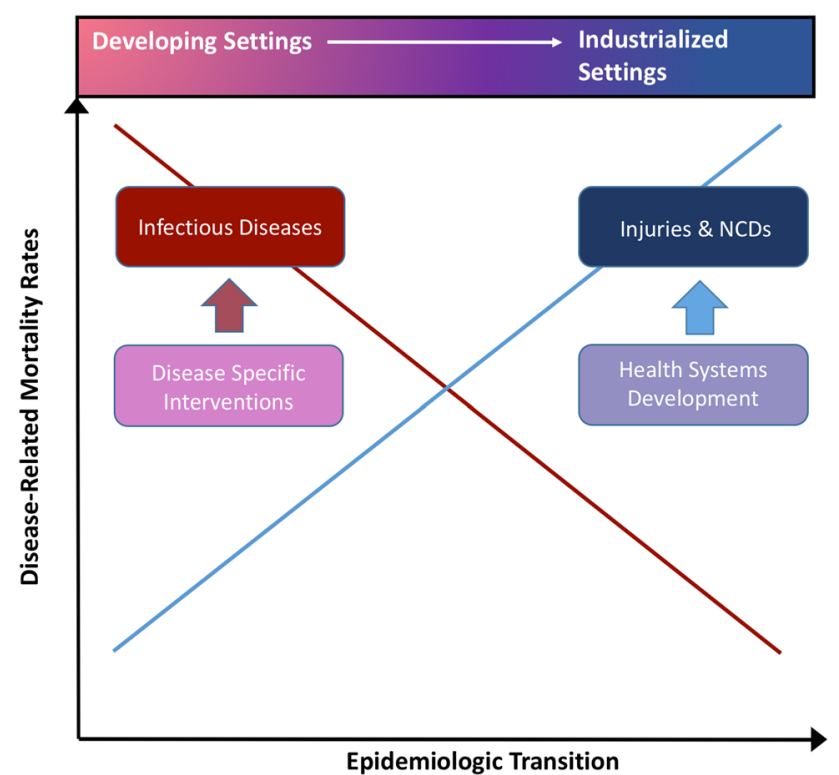

Fig. 1 Epidemiological transition in burden of disease: In developing settings, disease-specific interventions have led to decline in infectious disease; industrialization leads to predominance of noncommunicable diseases as well as injury. Health systems development is required to mitigate the harm of these conditions reframed and evaluated as an indication of health systems strength, (2) improvement of surgical systems by definition promotes creation of scalable health infrastructure, and (3) academic surgeons and societies should be leading global health systems development by promoting partnerships in research, education, training, and advocacy (Table 1).

\section{Position development}

The Society of University Surgeons' Committee on Global Academic Surgery developed this position as part of an initiative to foster the development of the field of academic global surgery. Discussion regarding surgical system strengthening was conducted among all invited and ad hoc members at serial Committee meetings to develop the tenets and ideas of the position. All drafts were shared among Committee members and underwent multiple rounds of edits based on Committee input until the position was reached and approved by the body.

\section{Surgical strengthening as health systems strengthening}

Patterns of disease in LMICs are changing rapidly, with increased prevalence of death and illness attributable to NCDs and injuries [2, 8]. However, the allocation of funding, services, and resources has not adapted with the changing epidemiology and remains largely focused on infectious disease priorities $[9,10]$. These resource disparities slow development of already underrepresented areas of healthcare [11]. Qualitative studies of funding and practice patterns in LMICs found that the abundant resources available for HIV led to a tendency for local physicians to "end up interpreting everything as related to HIV." [6] On a larger scale, donor directives shape the national health priorities of many LMICs. For example, despite low HIV prevalence, post-conflict Sierra Leone extensively developed HIV care delivery infrastructure using donor funds exclusively designated for HIV, while other health priorities were ignored [12]. Academic physicians in LMICs are incentivized toward specialties with increased funding for research and treatment [13], decreasing the availability of care in other subspecialties and perpetuating the cycle of imbalance. This diseasebased focus-fueled by development aid and donor pressure-reinforces the prevailing global health models of vertical programs often to the detriment of the overall health system.

The impact of such disease-specific "silver bullet" interventions is ultimately limited by weaknesses in the underlying health systems [11]. For example, health 
Table 1 Recommended pathway for horizontal health systems development in low- and middle-income countries through surgical systems strengthening

Reframe and evaluate surgical
delivery as an indication of
health systems strength
Building surgical capacity as a
horizontal approach to scaling
neglected medical areas
Championing of LMICs' health
systems development by
academic surgeons

\section{Recommendations}

- Adopt measures of surgical systems as proxies for overall health systems development

- Introduce interventions to improve the timely delivery of emergency surgical services

- Establish infrastructure for management of emergency surgical needs

- Recruit and train more health professionals

- Foster chronic disease management and long-term care

- Improve capacity to do elective procedures

Academic surgeons should lead efforts to promote and support research, education, training, and advocacy

\section{Research}

- Promote intramural and academic society funding for research and programs facilitating bilateral collaboration

- Facilitate training of LMIC physicians through fellowship grants

- Promote quality health systems development research

Education

- Prioritize education programs providing skills needed for health systems development

- Promote a multidisciplinary approach to medical education by integrating public health, biostatistics, quality control, and health economics

- Increase education materials and learning opportunities in LMIC settings

Leadership

- Foster mentorship between faculty and trainees at all levels

- Promote mentorship events at surgical meetings and within institutions

- Develop seminars on leadership development and government training in health systems

Advocacy

- Inform policy development

- Advocate for allocation of resources commensurate with burden of surgical disease

- Facilitate inclusion of LMIC members in US academic associations

\section{Collaboration}

- Establish long-term partnerships between HICs and LMICs to build research, education, and service delivery capacity

- Promote multidisciplinary collaborations

- Facilitate networking opportunities and academic exchanges

- Utilize academic institutions and associations to foster partnerships
Proposed actions

- Use existing surgical capacity assessment tools as surrogates to measure overall health system capacity

- Prioritize and finance emergency surgical services, services for pediatric surgical conditions, as well as the following elective surgical procedures: hernia repair, surgical treatment of cancer, and endocrine surgery

- Establish bidirectional exchange with LMIC trainees hosted in HIC settings

- Greater inclusivity by academic programs/ journals

- Academic institutions sharing e-libraries with their international partners

- Engage policymakers in LMICs in supporting research for surgical systems strengthening and translating findings into policy

- Conduct and support population-based surveys estimating the burden of diseases

- Encourage academic institutions and associations to foster partnerships with LMIC governments

LMICs low- and middle-income countries, HICs high-income countries 
programs that have poured resources into achieving longterm survival for patients with vertically transmitted HIV may have insufficient infrastructure to treat these same patients when they are injured in road traffic incidents or develop renal or cardiovascular disease from antiretroviral therapy.

The recent Ebola epidemic brought to public awareness of the interdependence of infectious disease with larger health systems. In Guinea, Sierra Leone, and Liberia, underdeveloped health care systems were incapable of mobilizing sufficient resources to mount an adequate response to the epidemic. Furthermore, the burden of addressing the crisis overwhelmed the already fragile health systems leaving no resources to manage other lifethreatening conditions such as non-Ebola infections and obstetric complications [14]. In contrast, Ebola was promptly and effectively contained in neighboring Nigeria, which had more robust health infrastructure and a higher per capita health care spending [15]. The difference in preparedness and outcomes in these settings highlights the vulnerability created by prioritizing vertical disease programming at the expense of horizontal development strategies.

There has been a widespread advocacy for developing capacity in underrepresented fields, in particular primary care [16], oncology [17] as well as injury management and elective general surgery [18]. Traditionally, surgical care was considered too resource intensive and costly to be considered a public health priority [19]. However, the Disease Control Priorities Project (DCPP) has repeatedly shown that effective surgical care impacts disability-adjusted life years (DALYs) with minimal cost [7]. However, critics have noted that continuing a "disease-specific" development approach is likely to leave systems vulnerable $[20,21]$. For these reasons, many have called for overall health systems development as a more balanced pathway to advancing global health. However, there remains ongoing debate regarding how best to implement systems strengthening.

The delivery of adequate surgical care requires integration of multiple areas of systems development, including infrastructure, personnel, equipment, and supplies [22]. The infrastructure needed to develop a "surgical ecosystem" [22] brings together many systems requirements of other specialty domains including obstetric, emergency, anesthesia, and primary care. Conversely, if a health system is unable to provide timely and adequate surgical care due to lack of one or more essential component(s), it is unlikely to provide adequate care in other domains. As such, we suggest surgical system development is a proxy for health systems development.

Several studies have developed survey-based instruments to assess surgical capacity and deficits in LMICs
[23-33]. Given the close relationship between surgical and overall health system development, we suggest that these tools could be utilized to assess overall health systems capacity and provide a valuable and cost-effective tool for evaluating development interventions.

\section{Surgical strengthening translates to other medical areas in need of development}

Building surgical capacity is by definition a "horizontal approach" and requires the development of the health system as a whole. In other fields, it is possible to increase access to medication or testing for a specific condition without otherwise changing health infrastructure. However, due to the complexity of the physical and human resources needed for the delivery of surgical care, surgical strengthening cannot develop in a silo. Development of surgical capacity functions as a gateway for systems development as many components of surgical care delivery are flexible and can be mobilized to treat other disease priorities.

- Emergency surgical services Interventions that improve timely delivery of urgent surgical care create infrastructure to treat other emergencies. Effective trauma and surgical emergency systems tailored to the unique epidemiology and structure of individual LMIC will require advanced pre-hospital and hospital emergency care coordination, as well as anesthesia and operative capacity. Once established, the core equipment, training, and protocols developed to triage and manage injuries can be adapted to improve care for other timesensitive medical emergencies such as maternal and obstetric emergencies and sepsis. Development of anesthesia and critical care capacities are essential for surgical care and translate to improved obstetric, pediatric, neonatal, and medical emergency care. Infrastructure development and development of multidisciplinary teams will also reduce delays and improve quality in the delivery of routine surgical care.

- Elective surgical services Treatment of elective surgical conditions such as hernias, resectable cancers, and goiters has the potential to improve functional outcomes for affected individuals and leads to increased productivity, supporting community and regional development. Additionally, development of elective surgical services strengthens multidisciplinary teams and enhances connections between surgeons and other clinicians. Development of interdisciplinary professional networks allows for greater clinical and professional support and increases the support base for advocacy efforts. Bolstering and organizing care 
networks can also provide capacity for management of chronic diseases which rely on many of the same collaborations. Finally, increasing elective surgical services will reduce the rate of surgical emergencies (for example, repairing hernias in an elective setting will prevent incarcerated hernia presentations) decreasing strain to the overall health system as emergency surgery is associated with higher complication rates, longer hospital stays, increased costs, and higher mortality rates.

\section{Health care systems strengthening in LMICs is the purview of the academic surgeon}

Historically, academic surgeons have played a critical role in advancing the study and implementation of health systems development in high-income countries (HIC). Early surgical luminaries such as Ernest Codman were responsible for setting the critical precedent of tracking patient outcomes [34]. In the 1970s and 1980s, academic surgeons called for and pioneered the standardization of trauma care and institution of regional trauma systems [35, 36], which have been demonstrated to decrease mortality and improve outcomes in multiple settings [37-40]. Academic surgeons were also early adopters of quality improvement measures [41, 42], implementing trauma outcomes review decades prior to the initiation of similar review by the broader medical community and formalization of Trauma Quality Improvement Programs (TQIPs) [43]. Ongoing examples of academic surgery leadership in health systems development include efforts to minimize preventable surgical errors by employing tools such as the Surgical Safety Checklist $[44,45]$ and standardizing methods for improving outcomes after surgery including implementation of the Surgical Care Improvement Project (SCIP) measures [46]. Thus far, however, this traditional systems-strengthening role of surgeons has been undervalued in global health.

The reliance and interdependence of surgical capacity and health systems development makes it logical and appropriate for academic surgeons to champion systems strengthening in LMICs, just as they have done in HICs. To facilitate, academic surgical societies and institutions in both settings should provide support to clinicians, researchers, and trainees interested in developing LMICs' health systems. This support should leverage each of the core competencies of academic surgery:

- Research This includes promotion of intramural and academic society funding for student and faculty research projects and programs that facilitate bilateral collaboration with LMIC partners and prioritize comprehensive systems strengthening. For example, the
Association for Academic Surgery (AAS) has instituted a global surgery fellowship grant for surgery trainees and offers exchange awards for conferences, facilitating training of LMIC physicians. Ideally, academic programs would promote bidirectional exchange with LMIC trainees hosted in HIC settings. Academic programs at regional and national academic conferences and in journals should be inclusive of quality research presenting data on health systems development. In LMICs where academic surgeons are often overburdened with clinical and teaching duties, collaboration with surgical societies could promote additional support and resources for research development, as well as a broader base for policy advocacy.

- Education Programs promoting health systems development skills should be prioritized at national and international meetings, with seminars appropriate for faculty within and outside the field. Education should heavily rely on multidisciplinary integration with experts in public health, biostatistics, quality control, and health economics and should be fundamentally grounded in collaboration with LMIC partners. Examples could include opportunities for observerships in American College of Surgeons Committee on Trauma for trauma professionals and participation/observership in TQIP/NSQIP outcomes policy creation. TQIP has already established several travel and research scholarships specifically directed at promoting education and development of international surgeons. In addition, formal partnerships between HIC and LMIC academic institutions could develop forums for electronic sharing of libraries, software, and other resources to promote education and research endeavors.

- Leadership Work is needed to establish health systems development as a widely accepted and well-delineated pathway in academic surgery. Career and academic milestones in this growing field will continue to be defined with academic bodies and may require shifts from canonical expectations of time allocation, clinical load, and approach to funding development for surgeons with careers focused on basic and translational research. Mentorship should be fostered between senior and junior faculty as well as between faculty and trainees by instituting and promoting both formal and informal/social mentorship events both at surgical meetings and within institutions. In LMICs, time constraints and lack of research funding are often critical barriers to research and publication, which are necessary for academic advancement. Collaboration with surgical societies can help to provide opportunities for funding, research, and training to allow for career development and promotion. Formal seminars on leadership development and governance training in 
health systems should be available for both future HIC and LMIC surgeon leaders. One example of this type of programming is the Brandeis Heller Leadership Program for Health Policy and Management which is an annual training program co-sponsored by the American College of Surgeons and the Thoracic Surgical Foundation that provides intensive practicums on policy and reform. Opportunities such as this one should be extended to LMIC partners.

- Advocacy A critical and often overlooked responsibility of academic surgeons is to inform policy development and advocate for allocation of resources commensurate with the burden of surgical disease. First steps may involve surveillance mechanisms capable of generating critical epidemiologic data, as in international trauma registries. If surgical system strengthening gains traction as a demonstrable means of improving the overall capacity of health systems, this would have policy implications at the regional, national, and international levels and serve as a leverage point for furthering the global surgery agenda both in terms of awareness and funding. Furthermore, by participating in academic surgical societies, LMIC and HIC surgeons have the potential to develop a critical base for advocating policymakers. Without establishing longitudinal relationships with policymakers, it will be difficult to establish buy-in for sustainable, impactful systems changes.

- Collaboration Global surgery has moved beyond shortterm mission trips and evolved into sustained commitments through HIC-LMIC partnerships designed to build capacity in research, education, and service delivery. Academic institutions and associations can play a critical role in fostering these partnerships, combining resources and expertise from both contexts to generate and implement innovative solutions. At the association level, AAS has supported and promoted international travel grants to facilitate exchange between its scholars and those from the West African College of Surgeons and the Colombian Surgical Association. Awards such as these allow scholars to attend each other's organizational annual meetings, facilitating rich networking opportunities and academic exchange. These collaborations would ideally be multidisciplinary and include ongoing relationships with academic anesthesia and nursing organizations.

\section{Conclusion}

Global public health is in a time of transition with increasing emphasis on health systems strengthening as a paradigm and priority in LMICs. As the state of surgical care is highly reflective of the maturity of the overall health systems, we advocate that investments in surgical systems in LMICs be considered a surrogate for health system strengthening. This will facilitate development of robust health care systems capable of comprehensive health care delivery well beyond surgical diseases. It is imperative that academic surgeons extend their historical leadership role in order to facilitate health systems building in resourceconstrained environments. Surgeons have been historical vanguards for health systems organization and strengthening but have not yet fully reprised this role in the global health domain. We call upon academic surgeons to prioritize building long-term sustainable research, clinical, and advocacy partnerships with LMIC surgical leaders and trainees to champion the sustainable development of health systems though surgical strengthening.

\section{Compliance with ethical standards}

Conflicts of interest All authors declare that they have no conflict of interest.

\section{References}

1. Dieleman JL, Schneider MT, Haakenstad A, Singh L, Sadat N, Birger M, Reynolds A, Templin T, Hamavid H, Chapin A, Murray CJ (2016) Development assistance for health: past trends, associations, and the future of international financial flows for health. Lancet 387(10037):2536-2544

2. Mortality GBD, Causes of Death C (2015) Global, regional, and national age-sex specific all-cause and cause-specific mortality for 240 causes of death, 1990-2013: a systematic analysis for the Global Burden of Disease Study 2013. Lancet 385(9963):117-171

3. Beaglehole R, Bonita R, Horton R, Adams C, Alleyne G, Asaria P, Baugh V, Bekedam H, Billo N, Casswell S, Cecchini M (2011) Priority actions for the non-communicable disease crisis. Lancet 377(9775): 1438-1447

4. Dalal S, Beunza JJ, Volmink J, Adebamowo C, Bajunirwe F, Njelekela M, Mozaffarian D, Fawzi W, Willett W, Adami HO, Holmes MD (2011) Non-communicable diseases in sub-Saharan Africa: what we know now. Int J Epidemiol 40(4):885-901

5. Chao TE, Sharma K, Mandigo M, Hagander L, Resch SC, Weiser TG, Meara JG (2014) Cost-effectiveness of surgery and its policy implications for global health: a systematic review and analysis. Lancet Glob Health 2(6):e334-e345

6. Grimes CE, Henry JA, Maraka J, Mkandawire NC, Cotton M (2014) Cost-effectiveness of surgery in low-and middle-income countries: a systematic review. World J Surg 38(1):252-263. https://doi.org/10.1007/s00268-013-2243-y 
7. Mock CN, Donkor P, Gawande A et al (2015) Essential surgery: key messages from Disease Control Priorities. Lancet 385(9983):2209-2219

8. Organization WH (2011) Global status report on noncommunicable diseases 2010. World Health Organzation, Geneva

9. Price R, Makasa E, Hollands M (2015) World Health Assembly Resolution WHA68. 15: "strengthening emergency and essential surgical care and anesthesia as a component of universal health coverage"-addressing the public health gaps arising from lack of safe, affordable and accessible surgical and anesthetic services. World J Surg 39(9):2115-2125. https://doi.org/10.1007/s00268015-3153-y

10. Dieleman JL, Graves CM, Templin T, Johnson E, Baral R, Leach-Kemon K, Haakenstad AM, Murray CJ (2014) Global health development assistance remained steady in 2013 but did not align with recipients' disease burden. Health Aff 33(5):878-886

11. Prince RJ, Otieno P (2014) In the shadowlands of global health: observations from health workers in Kenya. Glob Public Health 9(8):927-945

12. Benton A (2015) HIV exceptionalism: development through disease in Sierra Leone. University of Minnesota Press, Minneapolis

13. Good MJ, Mwaikambo E, Amayo E et al (1999) Clinical realities and moral dilemmas: contrasting perspectives from academic medicine in Kenya, Tanzania, and America. Daedalus 128(4):167-196

14. Marie-Paule Kieny DBE, Kadandale Sowmya (2014) Healthsystem resilience: reflections on the Ebola crises in western Africa. Bull World Health Organ 92:850

15. Ohuabunwo C, Ameh C, Oduyebo O et al (2016) Clinical profile and containment of the Ebola virus disease outbreak in two large West African cities, Nigeria, July-September 2014. Int J Infect Dis 53:23-29

16. Sixty-Second World Health Assembly. Resolution WHA62.12 (2009) Primary health care, including health system strengthening. http://www.who.int/hrh/resources/A62_12_EN.pdf. Accessed 17 Aug 2018

17. Farmer P, Frenk J, Knaul FM et al (2010) Expansion of cancer care and control in countries of low and middle income: a call to action. Lancet 376(9747):1186-1193

18. McQueen KA, Ozgediz D, Riviello R et al (2010) Essential surgery: integral to the right to health. Health Hum Rights 12(1):137-152

19. Ozgediz D, Jamison D, Cherian M et al (2008) The burden of surgical conditions and access to surgical care in low- and middle-income countries. Bull World Health Organ 86(8):646-647

20. Waage J, Banerji R, Campbell O et al (2010) The millennium development goals: a cross-sectoral analysis and principles for goal setting after 2015 Lancet and London International Development Centre Commission. Lancet 376(9745):991-1023

21. Feachem R, Yamey G, Schrade C (2010) A moment of truth for global health. BMJ 340:c2869

22. DeVries CR, Rosenberg JS (2016) Global surgical ecosystems: a need for systems strengthening. Ann Glob Health 82:605-613

23. Kushner AL, Cherian MN, Noel L et al (2010) Addressing the millennium development goals from a surgical perspective: essential surgery and anesthesia in 8 low- and middle-income countries. Arch Surg 145(2):154-159

24. Petroze RT, Nzayisenga A, Rusanganwa V, Ntakiyiruta G, Calland JF (2012) Comprehensive national analysis of emergency and essential surgical capacity in Rwanda. $\mathrm{Br} \mathrm{J}$ Surg 99(3):436-443

25. Kingham TP, Kamara TB, Cherian MN et al (2009) Quantifying surgical capacity in Sierra Leone: a guide for improving surgical care. Arch Surg 144(2):122-127 (discussion 128)
26. Tran TM, Saint-Fort M, Jose MD, Henrys JH, Pierre JBP, Cherian MN, Gosselin RA (2015) Estimation of surgery capacity in Haiti: nationwide survey of hospitals. World J Surg 39(9):2182-2190. https://doi.org/10.1007/s00268-015-3089-2

27. Linden AF, Sekidde FS, Galukande M, Knowlton LM, Chackungal S, McQueen KK (2012) Challenges of surgery in developing countries: a survey of surgical and anesthesia capacity in Uganda's public hospitals. World J Surg 36(5):1056-1065. https://doi.org/10.1007/s00268-012-1482-7

28. Iddriss A, Shivute N, Bickler S, Cole-Ceesay R, Jargo B, Abdullah F, Cherian M (2011) Emergency, anaesthetic and essential surgical capacity in the Gambia. Bull World Health Organ 89:565-572

29. LeBrun DG, Chackungal S, Chao TE, Knowlton LM, Linden AF, Notrica MR, Solis CV, McQueen KK (2014) Prioritizing essential surgery and safe anesthesia for the post-2015 development agenda: operative capacities of 78 district hospitals in 7 low-and middle-income countries. Surgery 155(3):365-373

30. Aboutanos MBMF, Rodas E, Salamea J, Parra MO, Salgado E (2010) Ratification of IATSIC/WHO's guidelines for essential trauma care assessment in the South American region. World J Surg 34(11):2735-2744. https://doi.org/10.1007/s00268-0100716-9

31. Carlson LC, Lin JA, Ameh EA, Mulwafu W, Donkor P, Derbew M, Rodas E, Mkandawire NC, Dhanaraj M, Yangni-Angate H, Sani R (2015) Moving from data collection to application: a systematic literature review of surgical capacity assessments and their applications. World J Surg 39(4):813-821. https://doi.org/ 10.1007/s00268-014-2938-8

32. Wong EG, Gupta S, Deckelbaum DL, Razek T, Kushner AL (2015) Prioritizing injury care: a review of trauma capacity in low and middle-income countries. J Surg Res 193(1):217-222

33. Blair KJ, Paladino L, Shaw PL, Shapiro MB, Nwomeh BC, Swaroop M (2017) Surgical and trauma care in low-and middleincome countries: a review of capacity assessments. J Surg Res 210:139-151

34. Codman NDEA (2002) Heroes and martyrs of quality and safety: Earnest Armory Codman MD. BMJ Qual Saf Health Care 11:104-105

35. American College of Surgeons Committee on Trauma (1976) Optimal hospital resources for care of the seriously injured. Bull Am Coll Surg 61(9):15-22

36. Shackford SR, Hollingworth-Fridlund P, Cooper GF et al (1986) The effect of regionalization upon the quality of trauma care as assessed by concurrent audit before and after institution of a trauma system: a preliminary report. J Trauma 26(9):812-820

37. Nathens AB, Jurkovich GJ, Rivara FP et al (2000) Effectiveness of state trauma systems in reducing injury-related mortality: a national evaluation. J Trauma 48(1):25-30 (discussion 30-21)

38. Shafi S, Nathens AB, Elliott AC et al (2006) Effect of trauma systems on motor vehicle occupant mortality: a comparison between states with and without a formal system. J Trauma 16(6):1374-1378 (discussion 1378-1379)

39. Cayten CG, Quervalu I, Agarwal N (1999) Fatality analysis reporting system demonstrates association between trauma system initiatives and decreasing death rates. $\mathrm{J}$ Trauma 46(5):751-755 (discussion 755-756)

40. Jurkovich GJ, Mock C (1999) Systematic review of trauma system effectiveness based on registry comparisons. J Trauma 47(3 Suppl):S46-S55

41. Shackford SR, Hollingsworth-Fridlund P, McArdle $M$ et al (1987) Assuring quality in a trauma system — the Medical Audit Committee: composition, cost, and results. J Trauma 27(8):866-875 
42. O'Keefe GE, Maier RV, Diehr P et al (1997) The complications of trauma and their associated costs in a level I trauma center. Arch Surg 132(8):920-924 (discussion 925)

43. Shafi S, Nathens AB, Cryer HG et al (2009) The trauma quality improvement program of the American College of Surgeons Committee on Trauma. J Am Coll Surg 209(4):521-530 e521

44. Haynes AB, Weiser TG, Berry WR et al (2009) A surgical safety checklist to reduce morbidity and mortality in a global population. N Engl J Med 360(5):491-499
45. Conley DM, Singer SJ, Edmondson L et al (2011) Effective surgical safety checklist implementation. J Am Coll Surg 212(5):873-879

46. Stulberg JJ, Delaney CP, Neuhauser DV et al (2010) Adherence to surgical care improvement project measures and the association with postoperative infections. JAMA 303(24):2479-2485 